ENCYCLOPEDDE Encyclopédie berbère

BERBERE

$20 \mid 1998$

20 | Gauda - Girrei

\title{
Ghât (Rhat)
}

(voir A134, Ajjer, E.B. VIII, p. 1268-1275)

E.B.

\section{OpenEdition}

Journals

Édition électronique

URL : http://journals.openedition.org/encyclopedieberbere/1921

DOI : 10.4000/encyclopedieberbere.1921

ISSN : 2262-7197

Éditeur

Peeters Publishers

Édition imprimée

Date de publication : 1 octobre 1998

Pagination : 3101-3107

ISBN : 2-7449-0028-1

ISSN : 1015-7344

Référence électronique

E.B., " Ghât (Rhat) », Encyclopédie berbère [En ligne], 20 | 1998, document G42, mis en ligne le 01 juin

2011, consulté le 25 septembre 2020. URL : http://journals.openedition.org/encyclopedieberbere/1921 ; DOI : https://doi.org/10.4000/encyclopedieberbere.1921

Ce document a été généré automatiquement le 25 septembre 2020.

(c) Tous droits réservés 


\section{Ghât (Rhat)}

(voir A134, Ajjer, E.B. VIII, p. 1268-1275)

E.B.

1 Ghât est située à l'est du Tassili n'Ajjer, à une vingtaine de kilomètres à vol d'oiseau de la frontière algérienne. L'oasis est dans la large vallée de l'oued Tannezrouft qui sépare le Tassili et la Tadrart. En amont, à huit kilomètres au sud de Ghât se situe, à El Barkat, une vaste nécropole protohistorique révèle l'antiquité de l'occupation humaine dans cette zone privilégiée; à quarante kilomètres plus au sud se trouve, à Tin Alkum, la même association d'un habitat actuel et d'une nécropole antérieure à l'Islam. D'autres témoignages plus anciens sont apportés par les gravures rupestres qui prolongent vers l'est la richesse artistique du Tassili n'Ajjer.

2 La ville de Ghât joue le rôle de capitale des Touaregs Ajjer (sur place on prononce Ajjir), mais d'une capitale qu'ils pressuraient en tout temps et en toute occasion. La frontière tracée entre la Libye et l'Algérie est totalement artificielle (la Tadrart n'est que le simple prolongement oriental du Tassili n'Ajjer), elle est issue du partage entre les possessions françaises à l'ouest et celle des Turcs puis des Italiens, à l'est. Ce partage est ignoré des Nomades en temps normal. La frontière a sectionné le territoire des Ajjer et réduit l'importance de Ghât à mesure que s'écroulait le commerce transsaharien au profit des villes du Fezzan (Mourzouk, Ghadamès...).

3 La première mention de Ghât est due à Ibn Battuta, au XIV siècle. Aucune donnée archéologique ne permet de remonter, au-delà. C'est en vain que B. Pace a proposé de retrouver le nom de la ville dans celui de Raspa, ville citée dans le triomphe de Cornélius Balbus en 20/21 av. J.-C. J. Desanges a montré que le proconsul n'avait guère dépassé au sud la capitale des Garamantes c'est à dire Ghadamès.

4 La ville resta sous la dépendance des Touaregs jusqu'au dernier tiers du XviII ${ }^{\mathrm{e}}$ siècle. Les sultans Imenanes, qui se disaient chérifiens et dont l'autorité s'étendait jusqu'aux Touaregs de l'Ahaggar, résidaient à Ghât. Vers 1660, une autre famille noble, les Urayen qui après avoir vécu au Fezzan, avait émigré au Soudan, à l'est de Tombouctou, renversa le sultan iménane Gouma, qui partageait sa résidence entre Djanet et Ghât. Gouma fut tué ainsi que son successeur Edukan et les Urayen s'arrogèrent le droit au tobol*. C'est aussi le moment où les Touaregs du nord se divisèrent en deux 
confédérations rivales, les Kel Ahaggar* et les Ajjer*. Cette division s'accompagna d'une rivalité permanente dont Ghât eut beaucoup à souffrir. Des récits, plus ou moins légendaires rapportent qu'à une date imprécise, dans le courant du XvIII ${ }^{\mathrm{e}}$ siècle, les Iménanes vengèrent la mort de Gouma grâce à une ruse. Un guerrier Iménane se présente au campement des Urayen et les défie au jeu de Kérad qui est proche du jeu de dames. Le défi ayant été relevé, l'Iménane se révéla être un joueur exceptionnel. Il gagna ainsi toutes les armes de ses adversaires, puis se retira après avoir demandé à ses hôtes de lui garder les armes réunies en faisceau dans une peau de bœuf détrempée. Il revint le lendemain en compagnie des guerriers iménanes qui massacrèrent leurs adversaires désarmés dont les lances et les épées étaient restées prisonnières du cuir durci. Ce récit donne une bonne image de ce que pouvaient être les rapports entre les clans touarègues rivaux.

Situation de la ville de Ghât.

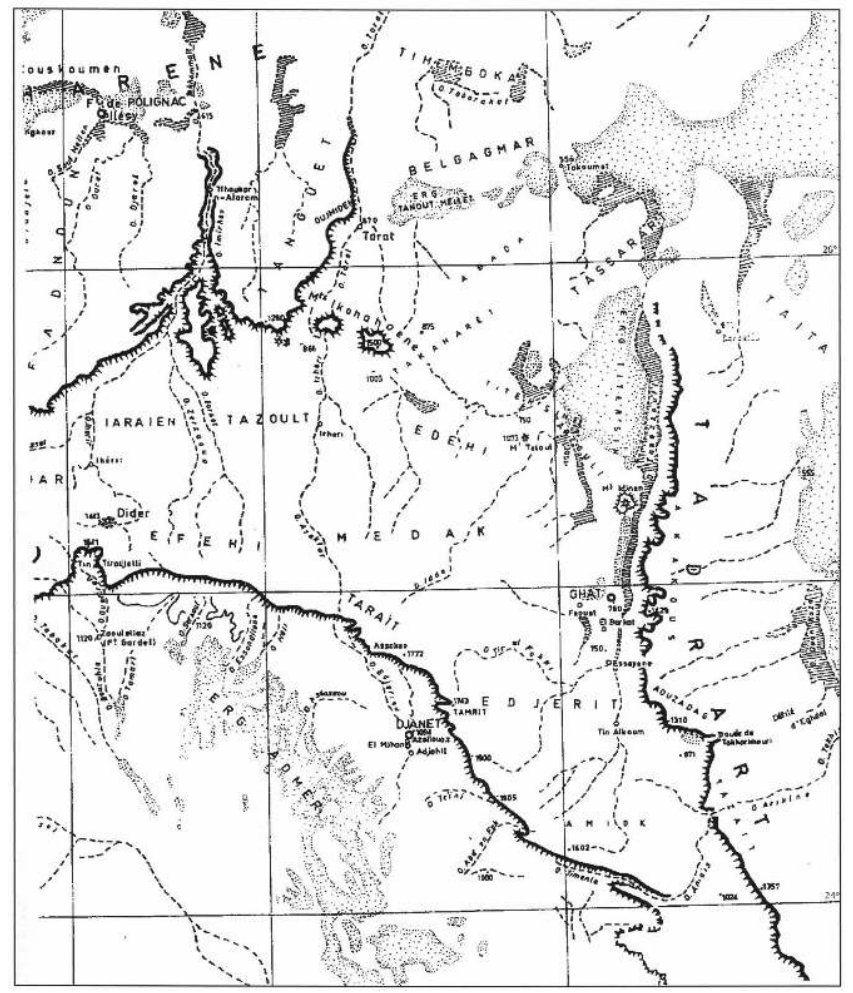

5 Vers 1800, le sultan de Mourzouk, maître du Fezzan, lance une expédition contre Ghât. La ville, défendue par les Urayen, résiste avec une telle opiniâtreté que l'ennemi abandonne le siège et perd dans l'affaire 200 dromadaires et les bagages qu'ils transportaient.

6 Aux querelles entre Touaregs, s'ajoute la lutte quasi permanente entre Chaamba et Ajjer : rezzou et contre-rezzou, embuscades, assassinats, pillages se multiplient tout le long $\mathrm{du} \mathrm{XIX}^{\mathrm{e}}$ siècle. Malgré cette insécurité, des voyageurs européens commencent à pénétrer dans ces terres cruelles et enveloppées de mystère. Le premier arrivé à Ghât venait d'une région proche : Richardson était le consul anglais de Ghadamès. En 1845, il entre à Ghât où il est bien accueilli. Fort de cette expérience, il revient cinq ans plus tard, à la tête d'une véritable mission qui comprenait deux scientifiques allemands : Henri Barth et Overweg. Au nom du gouvernement britannique, Richardson propose un 
traité aux autorités de la ville, par lequel les Ajjer renonceraient à la traite des esclaves. Les négociations n'aboutirent pas et les voyageurs s'enfoncèrent dans le désert. Richardson et Overweg devaient périr de maladie et d'épuisement mais Barth allait poursuivre son exploration pendant cinq années et en ramener une documentation d'une valeur exceptionnelle sur le Sahara et l'Afrique tropicale.

Onze ans plus tard, un autre voyageur, Henri Duveyrier se présente aux portes de Ghât ; bien qu'il bénéficie de la protection de la confrérie Tidjaniya qui étend son autorité sur une grande partie du Sahara, Duveyrier n'obtient pas l'autorisation de pénétrer en ville où les Senoussis construisent hâtivement leur zaouïa. Il put cependant rester quinze jours au pied des remparts et nous laisser une description de la ville.

D'octobre 1876 à janvier 1977, un autre explorateur germanique, Erwin de Bary, put séjourner à Ghât dont il donna une description pittoresque et plus précise que celle de Duveyrier. Son séjour à Ghât fut interrompu par une exploration du Tassili n'Ajjer et de la vallée de l'oued Imirou (qu'il nomme Mihero) où sont signalés des crocodiles. Il n'a pas la chance de voir ces reptiles mais il reconnaît un grand nombre de traces leur appartenant. E. de Bary se rendit ensuite en Aïr. De retour à Ghât, il meurt brusquement le lendemain de son arrivée (3 octobre 1878).

Une entrée de la ville ancienne (photo H. Claudot-Hawad).

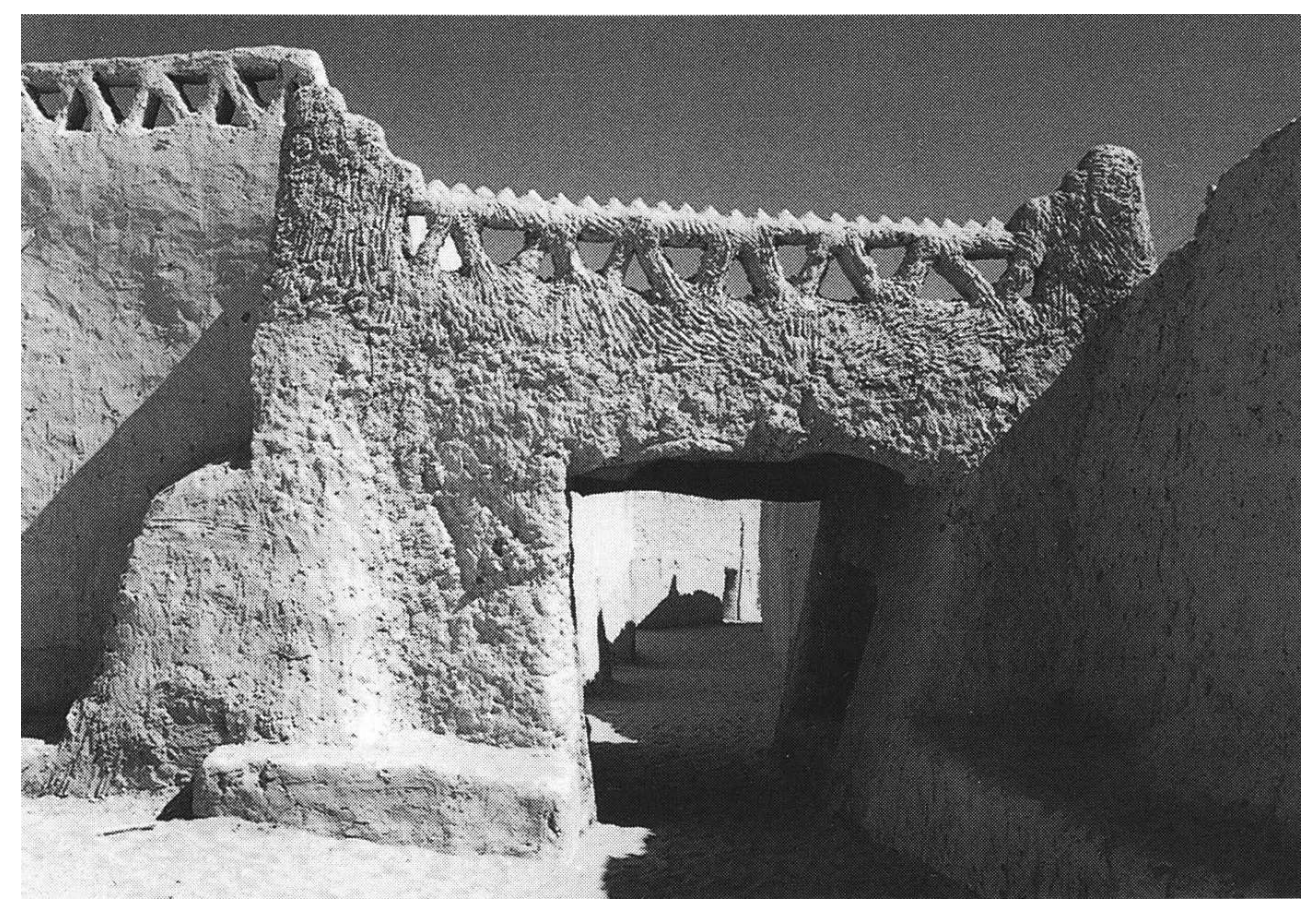

Pendant toute cette période qu'on pourrait nommer le protectorat touareg, Ghât est, théoriquement, sous l'autorité du sultan d'Istanbul qui est aussi Calife. La ville est gouvernée par un amyar*, le plus souvent berbère (Urayen, Imanyassaten, Ihadjenan) mais la politique suivie par les confréries (tidjaniya et surtout snoussiya) fait parfois confier l'administration de la ville à un "arabe". Tel fut le cas de El Hadj Ahmed ould Seddiq qui gouvernait Ghât en 1845 lors du premier voyage de Richarson. Une djemaa*, dont les membres étaient élus, servait de Conseil de gouvernement.

Tandis que Ghât attirait les voyageurs européens, les Turcs prenaient possession du Fezzan. Au même moment, à la demande même du nouveau gouverneur de la ville, El 
Hadj el Amin ould Seddiq, les Turcs sont invités à occuper la ville. Sur leur refus, El Amin veut se faire reconnaître comme vassal du Sultan. (1855). Riposte ou simple fait du hasard, c'est au cours de ces années que les Touaregs Ajjer assurent la protection d'une caravane "française" qui, partie d'Ouargla, arrive sans dommage à Ghât et en repart après quelques mois (1856-1858), retournant à son point de départ avec des présents destinés au gouverneur général de l'Algérie.

11 Le destin de Ghât n'est pas encore fixé. Il faut attendre l'année 1875 pour que la ville reçoive une garnison turque et renouvelle son acte d'allégeance à la Sublime Porte. A la suite de cette avancée, la willayat de Tripoli compte deux nouvelles circonscriptions, les caza de Bardaï et de Djanet. Ces deux agglomérations ont une situation géo-politique trop semblable pour qu'une telle décision soit le fait du hasard. Toutes deux sont des observatoires braqués sur des régions occupées par des populations difficiles, farouches et guerrières : Bardai fait face aux Toubous, comme Djanet surveille les Ajjer.

La ville dominée par le fort italien (photo H. Claudot-Hawad).

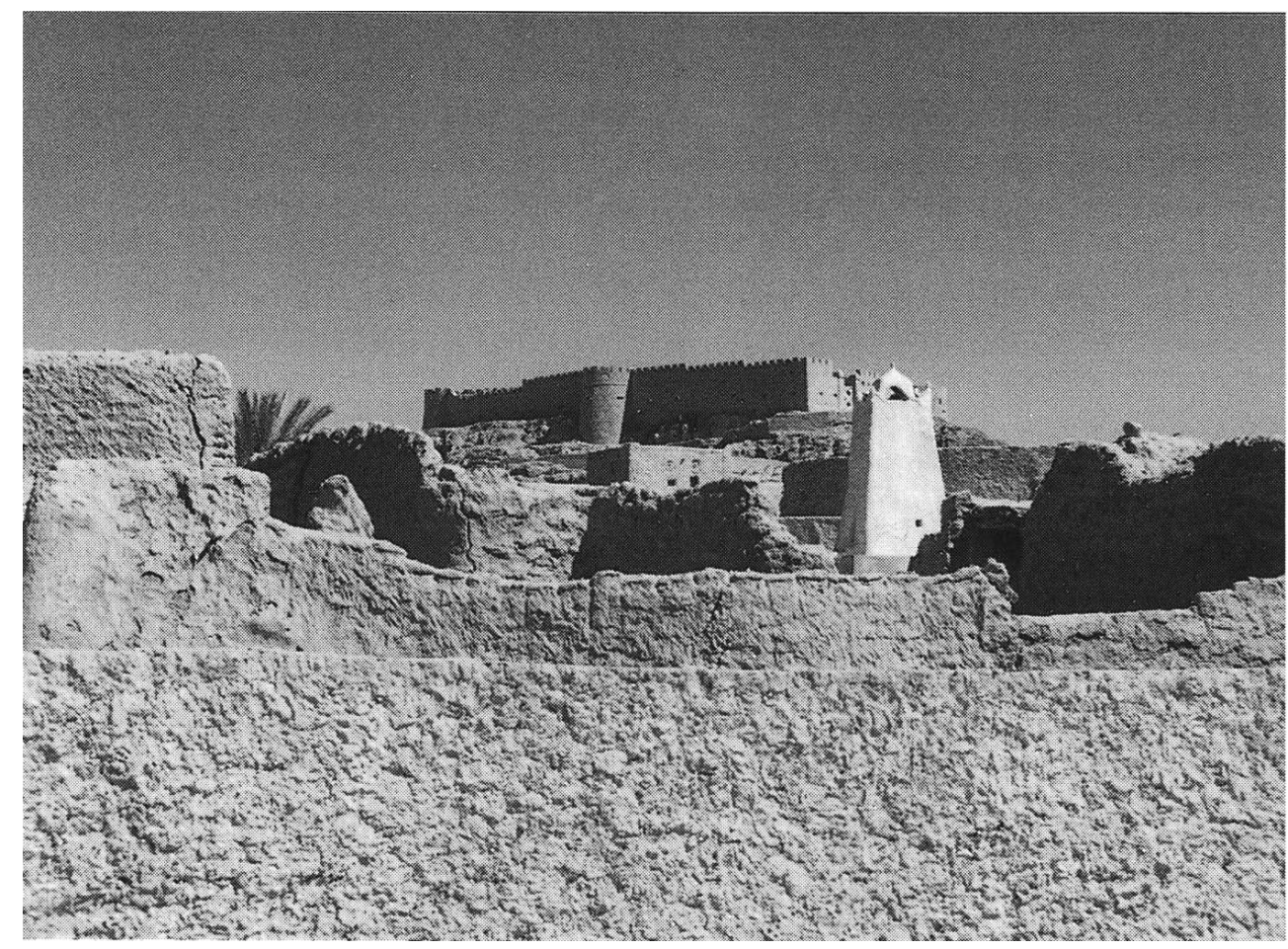

La domination turque et la présence effective des soldats du Sultan sont bien acceptées des Ghâti, ce n'est pas le cas des Touaregs qui auraient bien voulu recouvrer leur liberté d'action. Mais la seule tentative vraiment sérieuse fut le massacre des Turcs de Ghât en 1886. En 1905 arrive à Ghât sous le nom d'emprunt d'Abd el-Kader, Djami Bey exilé dans cette province lointaine pour ses idées politiques, ce qui n'empêcha point son accession au grade de kaïmakam dès l'année suivante (1906). Les Senoussi poursuivent leur action et multiplient leurs interventions ou proclamations contre les Français et ceux qui acceptent de les servir. La guerre italo-turque (1911-1912) puis la Grande Guerre de 1914-1918 eurent des conséquences imprévues au Sahara: en août 1914 les Italiens occupent Ghât, mais ils sont obligés de l'évacuer en octobre. C'est à la suite de dures campagnes et d'une guerre de conquête que les Italiens s'emparent à nouveau de Ghât en février 1930. Ils construisent la forteresse qui domine la vieille ville. En janvier 1943, 
Ghât tombe aux mains des méharistes français venus de Djanet, tandis que les oasis du Fezzân sont occupées par les forces françaises libres du général Leclerc. La domination française sur Ghât et le Fezzân dura jusqu'à l'application du traité franco-libyen du 10 août 1955 qui rattachait Ghât et le Fezzân à la Libye.

\section{Ghât d'aujourd'hui}

13 Jusqu'à ces deux dernières décennies Ghât avait peu changé d'aspect et gardait les caractères signalés dans les premières descriptions. Comme la plupart des agglomérations fezzanaises, le ksar de Ghât possède une enceinte en terre banchée, peu solide, qui dessine un rectangle irrégulier (alors que Duveyrier la croyait circulaire) de $700 \mathrm{~m}$ sur $500 \mathrm{~m}$. Cette enceinte munie de créneaux était percée de cinq portes. La ville est dominée par le fort italien en partie ruiné. Duveyrier et, quelques années plus tard E. de Bary décrivent dans les mêmes termes les maisons de Ghât, qui sont petites, n'ayant qu'un seul étage et construites toutes sur le même modèle : une skifa (vestibule allongé) permet de pénétrer dans une cour carrée sur laquelle donnent les chambres et les magasins. La cour est, avec la porte d'entrée, la seule source de lumière et d'aération. Ces maisons sont toutes semblables; même celle du Kaïmakam ne se distingue pas des autres demeures.

Depuis l'établissement de la Jamahiriya libyenne, une cité moderne a été construite à côté de la vieille ville, évacuée sur ordre. Vidée de ses habitants, la cité de terre n'est plus entretenue, l'enceinte s'éboule en plusieurs endroits, les maisons ne servent plus que de dépôts et certaines sont "squatérisées" par des émigrés venus des Etats sahéliens, tandis que d'autres sont devenues des ateliers où s'affairent des forgerons venus de l'Aïr. La place principale qui avait une belle architecture rythmée par des arcades a été rasée afin d'agrandir le marché !

15 Dans les constructions modernes de la Mdina Jdida, la ville nouvelle, l'usage du toub* est abandonné au profit des parpaings en ciment et le centre est desservi par des rues goudronnées éclairées à l'électricité. Mais cette ville nouvelle conserve un aspect inachevé que soulignent les chantiers abandonnés

16 Ghât était longtemps restée la capitale des Ajjer, autour de laquelle gravitaient les tribus touarègues depuis Amguid au nord-ouest jusqu'à Mourzouk à l'est. La politique de sédentarisation et le contrôle étroit exercé sur les nomades font peu à peu disparaître ce genre de vie. L'oasis ne connait plus qu'une vie ralentie bien que subsistent les cultures vivrières traditionnelles: dattiers, arbres fruitiers, blé, orge, sorgho, légumes et, de plus en plus, des cultures fourragères mais l'abaissement de la nappe phréatique, anciennement exploitée par des puits à traction animale ou à balancier, rendait précaire la plupart de ces productions avant que n'aient été effectués des forages profonds.

17 La population de Ghât s'élevait, en 1973, à 6924 personnes. Elle est d'origines diverses : Touaregs, "Arabes" du Fezzân, familles venues de Ghadamès, "Atara" descendants d'esclaves soudanais. La langue courante est une forme du tamahaq. 


\section{La fête du sel}

Cette population conserve, aussi bien à El Barkat qu'à Ghât le souvenir d'une cérémonie qui fut supprimée en 1954 par les autorités du nouvel Etat libyen. Il s'agit de la "fête du sel" qui se déroulait le 27 ramdhan. Les jeunes filles des deux localités se rassemblaient vêtues de leurs plus beaux atours et se donnaient une allure guerrière en croisant sur la poitrine leur longue ceinture, comme le font les méharistes avec leurs cartouchières. Chaque groupe était précédé de drapeaux et de musiciens et chaque participante était armée d'un bâton. Après avoir récolté le sel, les deux groupes se rapprochaient et simulaient un combat qui très vite prenait un aspect rythmique. Puis commençait, par deux ou trois vieilles femmes et en public, l'inspection des virginités. Les parents attachaient un grand intérêt à présenter leur fille pubère à cette visite traditionnelle. Cette cérémonie est très proche d'une autre festivité rapportée par Hérodote (IV, 180) qui avait lieu chez les Auses et les Machlyes, Libyens voisins du lac Triton (petite Syrte). D'abord les jeunes filles des deux peuplades simulaient un combat à coups de bâton et de jets de pierres qui dégénérait parfois jusqu'à des accidents mortels; celles qui mouraient à la suite des coups reçus étaient considérées comme de fausses vierges. On reconnaît dans l'une et l'autre fête, à vingt-quatre siècles d'intervalle, la même action pseudo-guerrière associée à la manifestation de la virginité.

La route moderne qui réunit Ghât à Tripoli favorise le désenclavement de cet oasis mais aussi un exode massif des cultivateurs. Cependant de nouvelles palmeraies sont plantées en dehors des périmètres anciens.

\section{BIBLIOGRAPHIE}

AGostini E. de, “La conca di Gat”, Boll, geogr. del Gov. della Tripolitania, n 5-6, 1933-34.

BARTH H., Voyages et découvertes dans l'Afrique septentrionale et centrale pendant les années 1849 à 1855.

Paris, A. Bonne, 1860.

BARY E. von, Le dernier rapport d'un Européen sur Ghât et les Touareg de l'Air (Journal de voyage 1876-77), traduit et annoté par H. Schirmer, Paris, Fischbacher, 1898.

BOUDERBA, “Voyage à Ghat”, Bull, de la Soc. géogr. de Paris, 1860.

BRULARD M., “N’Tchisent, liaison saharienne, n²5, 1957, p. 12-17.

DESANGES J., "Le triomphe de Cornelius Balbus (19 av. J.-C.)*”, Rev. africaine, t. CI, 1957, p. 5-43.

DUVEYRIER H., Les Touareg du nord, Paris, Challamel, 1864.

GARDEL G., Les Touareg Ajjer, Alger, Baconnier, 1961. 
FOUREAU F, Rapport sur ma mission chez les Touareg Azdjer (octobre 1893-mars 1894), Paris, Challamel, 1894.

MARTEL A., La Libye, 1835-1990. Essai de géopolitique historique, Paris, PUF, 1991.

INDEX

Mots-clés : Architecture, Fezzan, Nomadisme, Ville 